\title{
Macropinocytosis Exploitation by Cancers and Cancer Therapeutics
}

\author{
Kevin D. Ha, Scott M. Bidlingmaier and Bin Liu* \\ Department of Anesthesia, UCSF Helen Diller Family Comprehensive Cancer Center, University of California, San Francisco,
} San Francisco, CA, USA

Macropinocytosis has long been known as a primary method for cellular intake of fluid-phase and membrane-bound bulk cargo. This review seeks to re-examine the latest studies to emphasize how cancers exploit macropinocytosis to further their tumorigenesis, including details in how macropinocytosis can be adapted to serve diverse functions. Furthermore, this review will also cover the latest endeavors in targeting macropinocytosis as an avenue for novel therapeutics.

Keywords: macropinocytosis, receptor-dependent macropinocytosis, macropinosome, targeted cancer therapy, tumor selective internalization, altered membrane dynamics, neurodegenerative disease, pathogen entry

\section{OPEN ACCESS}

Edited by:

Hiroyuki Kabayama,

RIKEN Brain Science Institute, Japan

Reviewed by:

Paul Anthony Gleeson,

University of Melbourne, Australia

Rohan David Teasdale,

University of Queensland, Australia Ikuhiko Nakase,

Osaka Prefecture University, Japan

*Correspondence:

Bin Liu

bin.liu@ucsf.edu

Specialty section:

This article was submitted to Membrane Physiology and Membrane

Biophysics,

a section of the journal

Frontiers in Physiology

Received: 27 May 2016

Accepted: 22 August 2016

Published: 12 September 2016

Citation:

Ha KD, Bidlingmaier SM and Liu B (2016) Macropinocytosis Exploitation by Cancers and Cancer Therapeutics.

Front. Physiol. 7:381.

doi: 10.3389/fphys.2016.00381

\section{INTRODUCTION}

Macropinocytosis is an endocytic pathway that leads to internalization of large patches of plasma membrane along with extracellular fluid through irregularly formed vesicles called macropinosomes. When compared to endosomes originating from coated vesicles, macropinosomes are significantly larger by a factor of up to a thousand-fold (Hansen and Nichols, 2009). Despite observations from as early as the 1930s that cancer cells exhibit hallmarks of macropinocytosis (Lewis, 1937), macropinocytosis was largely viewed as a canonical pathway for bulk-phase endocytosis. However, recent advances in research lead to surprising, direct roles for macropinocytosis within tumorigenesis. Furthermore, increasing numbers of studies demonstrated that macropinocytosis might play broader roles ranging from neurodegenerative disease to pathogen infiltration into host cells. This review aims to shed light on how the various aspects of macropinocytosis may be exploited by both cancers and anti-cancer therapeutics.

\section{The Biomechanics of Membrane Ruffling and Macropinocytosis}

One of the hallmarks of macropinocytosis is its reliance on the formation of expansive membrane ruffles within the plasma membrane, which contrasts the canonical, endocytic pathways that depend on coat proteins such as clathrin and caveolin (Doherty and McMahon, 2009). The membrane ruffles facilitate formation of randomly sized vacuoles ranging from 0.2 to $5 \mu \mathrm{m}$ in size, which are up to 50 -fold larger than the average $0.1 \mu \mathrm{m}$ size exhibited in protein-coated vesicles (Hewlett et al., 1994). Comprehensive reviews are available that cover the biomechanical underpinnings of macropinocytosis in detail (Kerr and Teasdale, 2009; Lim and Gleeson, 2011). Membrane ruffling is initiated by rapid polymerization of branching of actin filaments (Mullins et al., 1998) and direct or indirect disruption of actin dynamics impacts macropinocytosis (Seastone et al., 2001; Innocenti et al., 2004). Spatial regulation of macropinocytosis can be aided by actin motor proteins, possibly by delivering vesicles and proteins to requisite sites of membrane ruffling (Brzeska et al., 2016). Other spatial and temporal regulators include the Rho superfamily of GTPases, such as Rac and Cdc42, along with lipid rafts and lipid components such as phosphoinositides and cholesterol, phosphatidylinositol phosphates (PIPs), their accompanying 
kinases (PIKs) and phosphatases (Grimmer et al., 2002; Mañes et al., 2003; Innocenti et al., 2004; Redelman-Sidi et al., 2013; Levin et al., 2015; Viaud et al., 2016). The roles of both phospholipids and phosphoinositides in not only macropinocytosis but within general endocytosis have long been investigated, with numerous, comprehensive reviews available (Simonsen et al., 2001; Bohdanowicz and Grinstein, 2013). Lastly, distinct forms of membrane ruffling may occur. In addition to traditional membrane ruffling, another type, circular dorsal ruffles, exhibits a single, transient wave of membrane ruffles from the edges of the dorsal membrane that then constricts into an annulus within a short time scale, is capable of internalizing $>50 \%$ of ligand-bound growth receptors within minutes (Orth et al., 2006). It remains unclear exactly why different forms of membrane ruffling occur and whether their ensuing macropinosomes obey similar biomechanics compared to macropinosomes originating from traditional forms of membrane ruffling.

Despite uncovering multiple proteins that partake in the signal transduction behind membrane ruffling, it remains unclear how membrane ruffling is regulated. Questions remain regarding how macropinocytosis can be specifically activated in response to certain stimuli within distinct cell populations. For example, macropinocytosis can have dramatically different effects on distinct cell types such as glioblastoma multiforme (GBM) and pancreatic cancer cells. When macropinocytosis is upregulated in GBM cells by a quinine-derivative chemical, Vacquinol-1, massive membrane ruffling and macropinocytosis lead to cell death through excessive vacuolization and deformations in the plasma membrane (Kitambi et al., 2014). On the other hand, pancreatic cancer cells develop increased macropinocytosis to enhance cancer progression (Commisso et al., 2013). Interestingly, Vacquinol-1 only induces massive macropinocytosis in GBM cells and not fibroblasts or neurons, implicating the need for specific protein players in macropinocytosis (Kitambi et al., 2014). These studies largely implicate an involvement of cell- and tissue-specific signal transduction pathways that may differentially regulate macropinocytosis to suit their differentiated functions. As discussed later in this review, tissuespecific effects on macropinocytosis can also be observed through modulation of various proteins. Current understanding of the biomechanics behind tissue-specific forms of macropinocytosis remains murky at best.

After the commencement of membrane ruffling, folds and buds of the plasma membrane can undergo fission to yield macropinosomes. This fission can be accommodated by the actin-associated protein, coronin, which helps form macropinocytic vesicles and dissociates shortly after vesicle fission from the plasma membrane (Hacker et al., 1997; BoseDasgupta et al., 2015). Depending on their origin and cell type, macropinosomes can either recycle back to the plasma membrane to recycle cell surface receptors or merge with the endosomal/lysosomal network (Schnatwinkel et al., 2004). The intracellular itineraries of macropinosomes may significantly overlap with clathrin-dependent, endocytic pathways, including mergers with early endosomes and the endosomal recycling complex (Donaldson et al., 2009). Multitudes of protein regulators define each step of intracellular vesicle trafficking and as a result, cell types with varying proteomic profiles exhibit diverse, intracellular trafficking functions. Nevertheless, it remains unclear how different cell types manage to achieve different, regulated forms of macropinocytosis and how they can specify the itineraries of macropinosomes.

\section{Confirming the Involvement of Macropinocytosis}

As with other forms of endocytosis, macropinocytosis can be microscopically observed with fluorescent molecules. This method typically has allowed full observation of the entire macropinocytosis pathway, beginning with the formation of large endocytic vesicles at the cell surface and until endosomal maturation, where intracellular vesicles coalesce with the lysosomal network. Fluid-phase, soluble proteins and sugars such as albumin and dextran, respectively, have served as useful, fluorescent markers specific for macropinocytosis (Racoosin and Swanson, 1992; Li et al., 2013). These molecules can help implicate an involvement of macropinocytosis by specifically colocalizing with novel, endocytosed cargo. Endocytosed cargo that largely colocalize with either albumin or dextran, instead of coated-vesicle cargo such as transferrin or transferrin receptors, typically suggest macropinocytosis involvement. Furthermore, solutes and receptors that have been associated with macropinocytosis can potentially be used as macropinocytic markers themselves. Examples of this include immunofluorescence against macropinocytosis-dependent cell-surface receptors, such as EGFR or EphA2, which have both been used to induce or observe macropinocytosis (Berger et al., 2012; Ha et al., 2014). Not only can fluorescent microscopy decipher macropinocytosis in vitro, but it can be additionally employed for in vivo observation of macropinocytosis, including within xenograft tumors in mice (Commisso et al., 2014).

Colocalization with macropinocytosis markers alone is usually not enough to support macropinocytosis involvement. In addition to colocalization, studies typically utilize pharmacological reagents that have specific impacts on macropinocytosis but not canonical, protein-coated endocytosis. In this fashion, macropinocytosis-specific markers continue to be internalized into cells while markers for canonical protein-coated endocytosis, such as transferrin, suffer from disrupted internalization. Cargos of interest that continue to internalize into cells and colocalize with macropinocytosisspecific markers despite pharmacological inhibition of canonical forms of endocytosis strongly implicate an involvement for macropinocytosis. Amilorides, for example, have been shown to inhibit macropinocytosis while leaving other coatdependent, endocytic pathways intact (West et al., 1989; Dowrick et al., 1993). Amiloride-based chemicals can inhibit $\mathrm{Na}^{+} / \mathrm{H}^{+}$ exchangers and consequently induce acidification at the plasma membrane, which interferes with the activation of Rac and Cdc42 GTPases and subsequent actin remodeling (Koivusalo et al., 2010). Chemicals that inhibit actin filament polymerization, such as cytochalasin D, also inhibit macropinocytosis (Schliwa, 
1982; Heuser, 1989). Inhibitors that disrupt signal transduction pathways that regulate membrane ruffling, such as inhibitors against PI3K or PIP5K, also disrupt macropinocytosis (Araki et al., 1996; Brown et al., 2001). Other chemicals can activate macropinocytosis, such as aluminum fluoride, which activates ADP ribosylation factor 6 (ARF6)-dependent macropinocytosis (Radhakrishna et al., 1996). Other comprehensive reviews are available that dissect the broad arsenal of pharmacological reagents available to interrogate the numerous forms of endocytic pathways (Ivanov, 2008). Primary disadvantages are commonly associated with pharmacological reagents, however. These typically include unknown, cellular side effects that often reduce selectivity against specific forms of endocytosis.

In many cases, selective disruption of specific proteins may lead to fewer side effects in dissecting the multiple endocytic routes. Another review covers many protein and lipid regulators that can be overexpressed, ablated, or mutated to yield distinct effects on specific forms of endocytosis, including macropinocytosis (Amyere et al., 2002). Depending on the both the protein target and the severity of disruption, non-specific side effects can be somewhat controlled.

In summary, fluorescent microscopy, pharmacological disruption, and altered protein expression can all be employed to confirm specific involvement of macropinocytosis vs. canonical, endocytic pathways. The studies referenced throughout this review largely utilize combinations of these methods to suggest macropinocytosis involvement.

\section{Activation of Macropinocytosis through Receptors and Ligands}

Depending on the cell type, macropinocytosis can be primarily constitutive or induced. However, cells can commonly possess both forms of macropinocytosis. Furthermore, varying cell types can display wide ranges in macropinocytic activity. For example, macrophages and dendritic cells often utilize high levels of constitutive macropinocytosis to screen the extracellular environment for pathogenic materials (Kerr and Teasdale, 2009). On the other hand, neutrophils exhibit increased levels of macropinocytosis when sensing foreign pathogens, which might also aid in their ability to ingest pathogens (Lim and Gleeson, 2011).

Macropinocytosis is often mediated through stimulation of cell surface receptors. These include receptor tyrosine kinase (RTK) family receptors, where exosomes shed by cancer cells can internalize into distant cancer cells in a paracrine fashion through binding with RTKs (Li et al., 2010; Koumakpayi et al., 2011; Nakase et al., 2015), cell-surface proteoglycans which macropinocytose ligands containing closely spaced, basic amino acids such as lysine, arginine, and histidine (Magzoub et al., 2006), and G-protein coupled receptors (GPCRs). Podocytes and colorectal cancer cells both activate macropinocytosis through interactions between albumin-associated free fatty acids (FFAs) and GPCRs (Wu et al., 2013; Chung et al., 2015). Dendritic cells activate macropinocytosis through another GPCR, S1P receptors, to detect S1P and activate macropinocytosis (Ocaña-Morgner et al., 2011). Certain cell types, including murine bone marrow macrophages, do not require macropinocytosis stimulation and instead have constitutive macropinocytosis (Norbury et al., 1995).

Pathogens commonly display surface glycans to trigger macropinocytosis through interactions with C-type lectin receptors on antigen-presenting cells (Frenz et al., 2015). In other instances, certain receptors bind to non-protein ligands to activate macropinocytosis. For example, calcium is necessary for inducing constitutive macropinocytosis in cells through Gprotein-coupled calcium-sensing receptors (Canton et al., 2016). RTKs can also activate macropinocytosis through downstream activation of a small GTPase, Rab5, to induce circular membrane ruffles (Lanzetti et al., 2004).

Studies have demonstrated that proteins rich in arginine amino acids may internalize into cells specifically via macropinocytosis, possibly through basic interactions that results in crosslinking (Nakase et al., 2004). Indeed, a 12-mer arginine peptide, but not an 8-mer, induces macropinocytosis by cross-linking CXCR4, the co-receptor for HIV-1 infection into host cells (Tanaka et al., 2012). Additionally, crosslinking the complement receptor, CD46, either via antibodies or through pathogens such as the measles virus, induces macropinocytosis (Crimeen-Irwin et al., 2003). Receptor crosslinking and macropinocytosis activation may be more generic than previously thought, as studies have shown that receptor cross-linking enhances endocytosis (Moody et al., 2015).

\section{Cancers Exploit Macropinocytosis to Enhance Tumorigenesis}

Numerous studies have firmly established concrete roles for Rac in broad aspects of tumorigenesis, including cell survival and growth, metastasis, and secondary tumor establishment (Mack et al., 2011). Although Rac plays an important role as an upstream activator of macropinocytosis, it is difficult to determine whether macropinocytosis itself plays direct roles in the tumorigenic functions of Rac. Rac can be activated by members of the Ras (rat sarcoma) GTPase superfamily, which has long been heralded as the most frequently mutated family of genes in cancer (Stephen et al., 2014). Certain proteins, such as Abi1, can regulate both macropinocytosis and protein coat-dependent endocytosis and therefore it is likely that cancer cells can exploit multiple endocytic pathways to establish and maintain their oncogenic phenotypes (Innocenti et al., 2005).

Macropinocytosis serves as an efficient method to internalize cell surface receptors, which cancer cells can exploit in their favor. Cancers down-regulate cell-surface death receptors (DRs) via macropinocytosis in an H-Ras dependent manner to evade TNFrelated apoptosis-inducing ligands (TRAILs) and subsequent apoptosis (Chen et al., 2014). Interestingly, certain cancers with either K- or H-Ras mutations can still be sensitive to TRAILs as other receptors and downstream signaling components may affect DR internalization via macropinocytosis (Drosopoulos et al., 2005; Wang et al., 2015). Interestingly, cancers can utilize macropinocytosis to internalize and activate receptors. Prostate and breast cancer cells utilize macropinocytosis to translocate a growth factor receptor, ErbB3, from the plasma 
membrane into the nucleus to further cellular proliferation (Koumakpayi et al., 2011; Reif et al., 2016). It remains unclear exactly how a membrane-bound receptor, possibly within a vesicle, is imported into the nucleus. Additionally, H-Rastransformed fibroblasts internalize cell-surface platelet-derived growth factor $\beta$-receptors (PDGFR $\beta$ ) through macropinocytosis to sensitize and enhance PDGFR $\beta$ activation, resulting in increased anchorage-independent proliferation (Schmees et al., 2012). In this case, PDFGFR $\beta$ phosphorylation is enhanced only within macropinosomes, possibly due to macropinosomesspecific PI3K activity (Schmees et al., 2012).

As macropinocytosis is an efficient and rapid form of endocytosis, it is not surprising that cancers have exploited it to replenish scarce nutrients for sustained propagation within the tumor microenvironment. Ras-transformed pancreatic cancer cells can upregulate macropinocytosis to internalize and degrade albumin as a source of glutamine, which is one of the most deprived metabolites within tumor microenvironments (Commisso et al., 2013; Kamphorst et al., 2015). Macropinocytosis appears to be the key player in this phenomenon, as amiloride-based drugs that selectively inhibit macropinocytosis while leaving other coat-dependent endocytosis intact halted intake of albumin as a source of glutamine for cancer cells (Commisso et al., 2013). In a similar fashion to absorbing extracellular albumin, cancer cells have also been shown to internalize extracellular ATP to aid in cancer metabolism (Qian et al., 2014).

In addition to accumulating fluid-phase nutrients such as proteins and ATP, cancer cells often internalize secreted vesicles, called exosomes or microvesicles, through macropinocytosis (Nakase et al., 2015). Exosomes can carry proteins, lipids, and nucleic acids to serve as a form of extracellular communication or metabolic replenishment (Tkach and Thery, 2016). Many cancer types commonly exploit exosomes to enhance cancer progression, typically by secreting exosomes that internalize into other cancer cells and lead to favorable environments that promote angiogenesis, metastasis, and immunosuppression (Whiteside, 2016). For pancreatic cancer cells, internalizing exosomes requires K-Ras and EGFRdependent macropinocytosis (Nakase et al., 2015). Alternatively, exosomes originating from cancer cells can cause dysfunction in normal cells. For example, pancreatic cancer cells shed exosomes that can be internalized by $\beta$-cells to negatively impact insulin secretion and further pancreatic cancers (Javeed et al., 2015). Other cell types, such as cancer-associated fibroblasts, do not require oncogenic K-Ras signaling to internalize exosomes (Zhao et al., 2016).

\section{Disrupting Macropinocytic Biochemistry for Therapeutic Gains}

Established links between macropinocytosis and cancers have spurred the development of therapeutics targeting the biochemical regulation behind macropinocytosis. This includes therapeutics against the phosphoinositide biochemical pathways. For example, RNA interference (RNAi) of one particular PIP5K, I $\gamma$ i2, leads to decreased oncogenic growth of breast cancer cells
(Thapa et al., 2013). In this case, I $\gamma \mathrm{i} 2$ coordinates with Src to promote anchorage-independent growth. PIP5K may serve divergent functions across multiple tissue types. For example, disruption of PIP5K $\alpha$ stunted membrane and protein recycling from macropinosomes to the plasma membrane (Brown et al., 2001). It remains unclear whether membrane and protein recycling from macropinosomes contributes to anchorageindependent growth in breast cancer cells. In another example, the fungal metabolite wortmannin can block phosphoinositide3-kinase (PI3K) to inhibit scission of macropinosomes from the cell surface, thus negatively effecting pancreatic cancer motility, invasion, and metastasis (Araki et al., 1996; Teranishi et al., 2009). Future studies investigating overlapping functions across various phosphoinositide pathways within the context of macropinocytosis across multiple cancer cell types can potentially uncover additional therapeutic opportunities.

Another therapeutic venture includes disrupting cancer cell metabolic activity through inhibition of macropinocytosis (Zeitouni et al., 2016). However, cancer cells may additionally employ other compensatory methods such as membrane transporters and coat-dependent endocytosis to import extracellular nutrients and proteins (Selwan et al., 2016). Indeed, macropinocytosis can activate mTORC1 through nutrient-intake in cancer cells that rely on metabolizing endocytosed proteins in scarce amino-acid conditions (Palm et al., 2015; Yoshida et al., 2015). Macropinosomes eventually mature into increasingly acidic vacuoles, or lysosomes, for subsequent degradation of consumed proteins (Racoosin and Swanson, 1993). Hydroxychloroquine (HCQ), which inhibits lysosomal acidification, negatively impacts both autophagy and macropinocytosis-dependent scavenging and is currently under investigation for its cancer therapeutic potential (Wolpin et al., 2014). In this context, HCQ can be used to prevent cancer cells from breaking down extracellular proteins to generate metabolic substrates, thereby placing limitations on cancer cell metabolism and growth (Kimmelman, 2015).

Lastly, other therapeutic ventures have shown that hyperstimulating macropinocytosis in cancer cells can lead to nonapoptotic cell death known as methuosis (Li et al., 2010; Maltese and Overmeyer, 2015). Methuosis can be induced through hyperactivated Ras in glioblastoma cells, contributing to excessively large macropinosomes that ultimately result in cell rupture (Overmeyer et al., 2008). As Ras is commonly upregulated in numerous cancers, glioblastoma cells must lack certain signal transduction pathways that regulate macropinocytosis levels. Uncovering these pathways, if they exist, should help expand the arsenal of available targets in disrupting macropinocytosis.

\section{Exploiting Macropinocytosis for Targeted Delivery of Anti-Cancer Therapeutics}

As cancer cells frequently employ macropinocytosis to aid in receptor regulation and internalize essential metabolites, extensive efforts have been underway in utilizing macropinocytosis to deliver cytotoxic therapeutics specifically into cancer cells. Some anti-cancer agents innately undergo macropinocytosis, such as AS1411, which internalizes into 
various cancer cells through cell-surface nucleolin-dependent mechanisms that activate macropinocytosis only in malignant cells (Reyes-Reyes et al., 2010). Other therapeutics specifically target cell surface receptors that may trigger macropinocytosis. For example, therapeutic drugs conjugated with peptides that target a combination of proteoglycans and keratinocyte growth factor receptors (KGFR) can selectively internalize into and kill KGFR-expressing lung cancer cells via macropinocytosis (Iglesias and Koria, 2015).

Potent cancer therapeutics that exhibit excessive, systemic toxicities or unstable pharmacokinetics from hydrophobic profiles are excellent candidates for conjugation to yield chemical conjugates or nanoparticles. The resultant conjugates or nanoparticles can be engineered to yield greater specificity and enhanced pharmacokinetics. In many other examples, synthetic conjugates comprising any combination of small chemicals, lipids, proteins, genetic components, and chemical scaffolds can be developed to form nanoparticles that can then be internalized into target cells via macropinocytosis. Nanoparticles, which vary widely in composition, size, chemical charge, and shape, all contribute to distinct, cellular specificities and in endocytic mechanisms (Kettler et al., 2014). Larger nanoparticles rely on macropinocytosis for efficient internalization but smaller nanoparticles can traffic into cells through proteincoated endocytosis as well. For example, nanoparticles featuring paclitaxel fused to a chimeric peptide comprising an elastinlike peptide with a hydrophilic peptide (CP-PTX) serve double purposes in reducing the hydrophobic profile of paclitaxel and in enhancing tumorigenic internalization of paclitaxel via macropinocytosis (Bhattacharyya et al., 2015; Iglesias and Koria, 2015). Nanoparticles can also deliver genetic materials. For example, polyethylene glycol (PEG)-based nanoparticles were successfully used to deliver genes into cancer cells through macropinocytosis (Walsh et al., 2006). Nanoparticle shapes play important roles as well, as rod-like nanoparticles internalize into cells independently from macropinocytosis (Liu et al., 2016). Nanoparticles can also be targeted against specific cell types by incorporating aptamers into lipid nanoparticles (Liang et al., 2015). Similarly, nanoparticles conjugated with antibodies targeting the collagen receptor, $\alpha 2 \beta 1$ integrin, lead to efficient internalization via macropinocytosis (Kankaanpää et al., 2015). As $\alpha 2 \beta 1$ integrins have been previously implicated in both cancer stem cells and tumor angiogenesis, $\alpha 2 \beta 1$ integrin-targeting nanoparticles could yield specific utility in cancer therapeutics (Naci et al., 2015).

Another intriguing therapeutic front includes conjugating cytotoxic payloads onto albumin primarily for enhancing drug pharmacokinetics and because albumin has long been observed to accumulate within solid tumors through macropinocytosis (Kratz, 2008). An example includes the FDA-approved, nanoparticle albumin-bound form of paclitaxel (nab-paclitaxel or Abraxane ${ }^{\circledR}$ ) for treating multiple cancers. However, cancers are still able to overcome these drugs through acquired resistance, likely in differentially expressed proteins that regulate macropinocytosis, including cytoskeletal and lipid metabolism proteins (Zhao et al., 2015b) or through increased drug exporters such as P-glycoprotein (Zhao et al., 2015a). Other albumin-based conjugates targeting folate receptors also demonstrated efficient delivery of cytotoxic compounds specifically into cancer cells (Shi et al., 2014). Conjugates with non-albumin carriers have also been successful, including paclitaxel poliglumex (PPX or formerly Xyotax), comprising paclitaxel and polyglutamic acid polymers. PPX has been effective against metastatic breast cancers when used in combination with capecitabine (Northfelt et al., 2014).

Another component commonly used for conjugation is the poly-arginine peptide. As poly-arginine peptides induce macropinocytosis and cancer cells generally have increased macropinocytosis, efforts have been underway in conjugating poly-arginine peptides with cytotoxic compounds. These conjugates can be employed to deliver a variety of materials into cells, including cytotoxic reagents against cancer cells (Biswas et al., 2013; Liu et al., 2014), genetic materials (Zhang et al., 2006; Hayashi et al., 2012), insulin (Liu et al., 2012; Zhang et al., 2015), and liposomes across the blood brain barrier (Qin et al., 2012). Interestingly, at least in HeLa cancer cells, polyarginine peptide entry into the cell cytosol occurs independently from macropinocytosis (Zaro et al., 2006). This suggests that poly-arginine peptides endocytosis may not be exclusive to macropinocytosis, depending on both the cargo and cell type.

As cancers routinely internalize exosomes, a logical maneuver includes formulating therapeutics that mimic exosomes. Exosomes can be derived from same-host cells to avoid inducing immune responses (Hall et al., 2016). Alternatively, exosomes originating from dendritic cells may help elicit immune responses by $\mathrm{T}$ and NK cells to specifically target cancer cells (Pitt et al., 2016). Exosomes can be prepared in vitro, with therapeutic proteins either electroporated into exosomes or intracellularly incorporated through targeted overexpression of genes in the cell cultures (Munson and Shukla, 2015). Furthermore, lipid-membrane coat-based delivery systems that resemble exosomes can be engineered in ways that promote endosomal escape after macropinocytic entry into cells to enable gene delivery into cells while avoiding degradation through the lysosomal pathway (Khalil et al., 2007). Exosomes are extremely useful in delivering payloads into cancer cells without requiring advanced chemical conjugation as often required in nanoparticles. Additionally, exosomes also offer greater flexibility in packaging diverse combinations of cargoes.

\section{Macropinocytosis in Pathogen-Host Interaction and Neurodegenerative Diseases}

If cancers commonly exploit macropinocytosis for efficient endocytosis of scarce nutrients and proteins, it is not surprising that various pathogens also infiltrate into host cells through this pathway. Pathogens such as viruses and bacteria commonly enter human cells by activating macropinocytosis through receptor-dependent means. Several examples include human cytolomegavirus (Hetzenecker et al., 2016), Mycobacterium smegmatis (Baltierra-Uribe et al., 2014), influenza A virus (de Vries et al., 2011), vaccinia virus (Mercer and Helenius, 2008), infectious bursal disease virus (Gimenez et al., 2015), 
Salmonella (Francis et al., 1993; Chen et al., 1996), Zaire Ebola virus (Hunt et al., 2011), and Kaposi's sarcoma-associated herpesvirus (Chakraborty et al., 2012). However, pathogens may enter cells through additional endocytic routes in addition to macropinocytosis. For example, African swine fever virus can internalize into macrophages through either macropinocytosis or clathrin-mediated endocytosis (Hernáez et al., 2016). Organisms that are larger than bacteria and viruses, such as protozoa, can also infiltrate into host cells through macropinocytosis (de Carvalho et al., 2015). In addition, Plasmodium sporozoites, or malaria, also infiltrate into host hepatocytes by inducing macropinocytosis through the EphA2 receptor (Kaushansky et al., 2015), possibly through receptor cross-linking (Ha et al., 2014).

Macropinocytosis appears to play vital roles across a variety of neurodegenerative diseases as well, and this has been comprehensively reviewed (Zeineddine and Yerbury, 2015). These diseases include Parkinson's disease, Huntington's disease, amyotrophic lateral sclerosis, and Alzheimer's disease. Macropinocytosis allows neuronal cells to internalize protein aggregates, possibly through cross-linking receptors that remain largely unknown. Cross-linking of amyloid precursor protein (APP) at the cell surface by antibodies leads to APP internalization via Arf6-depedent macropinocytosis, potentially

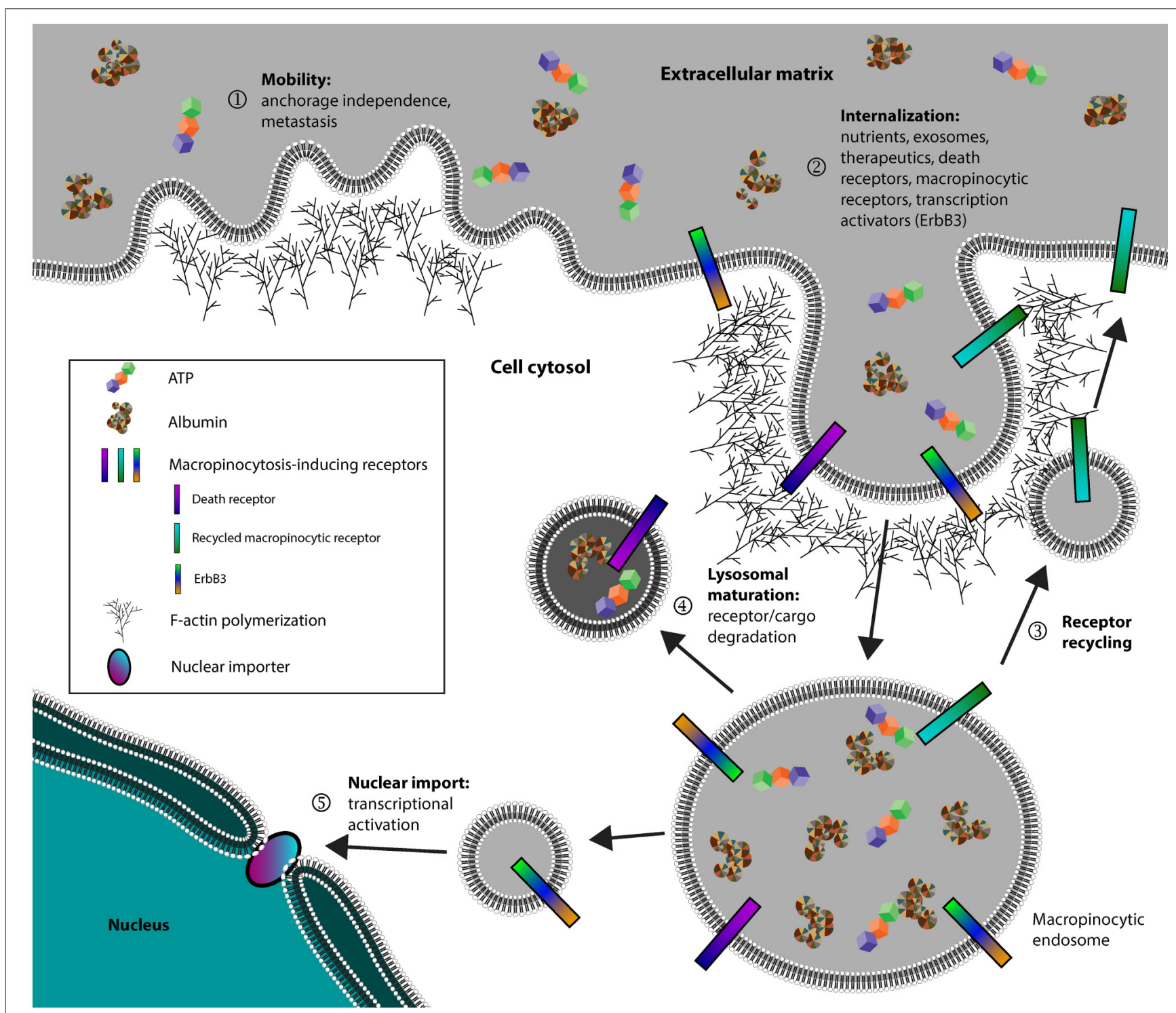

FIGURE 1 | Cancer cells exploit macropinocytosis to fulfill diverse functions. (1) Cancer cells can utilize membrane ruffles, a hallmark of macropinocytosis, to establish mobility and facilitate both anchorage independence and metastasis. (2) Cancer cells internalize metabolic nutrients, surface receptors, and transcription activators. (3) Receptors involved in activating macropinocytosis are recycled back to the plasma membrane. (4) Non-recycled protein cargo and receptors are degraded through the lysosomal network or in some instances, (5) certain receptors such as ErbB3 can be targeted for nuclear import to activate transcription. 
contributing to Alzheimer's disease (Tang et al., 2015). In some of these cases, heparin sulfate proteoglycans may serve as the receptors for internalizing tau and $\alpha$-synuclein fibrils to commence intracellular fibrillization (Holmes et al., 2013). As neurodegenerative fibrils commonly utilize macropinocytosis to infiltrate neuronal cells, therapeutics that specifically inhibit macropinocytosis in neurons sound logically promising. However, such therapeutics are currently not available due to the lack of knowledge in the signal transduction pathways that define both tissue-specific and universal aspects of macropinocytosis regulation. For example, therapeutics that exhibit strong, inhibitory effects against macropinocytosis may yield unintended side effects in disrupting constitutive macropinocytosis of both macrophages and dendritic cells.

\section{CONCLUSIONS}

Cancer cells, in addition to pathogens and neurodegenerative diseases, all exploit macropinocytosis for its efficiency in endocytosis. In particular, cancer cells seem to possess increased macropinocytic activity to fulfill diverse functions that include metastasis, metabolism, and signal transduction (Figure 1). For this reason, there has been an increased interest in the field of macropinocytosis-dependent therapeutics, expanding the

\section{REFERENCES}

Amyere, M., Mettlen, M., Van Der Smissen, P., Platek, A., Payrastre, B., Veithen, A., et al. (2002). Origin, originality, functions, subversions and molecular signalling of macropinocytosis. Int. J. Med. Microbiol. 291, 487-494. doi: 10.1078/14384221-00157

Araki, N., Johnson, M. T., and Swanson, J. A. (1996). A role for phosphoinositide 3-kinase in the completion of macropinocytosis and phagocytosis by macrophages. J. Cell Biol. 135, 1249-1260. doi: 10.1083/jcb.135. 5.1249

Baltierra-Uribe, S. L., García-Vásquez Mde, J., Castrejón-Jiménez, N. S., EstrellaPiñón, M. P., Luna-Herrera, J., and Garcia-Perez, B. E. (2014). Mycobacteria entry and trafficking into endothelial cells. Can. J. Microbiol. 60, 569-577. doi: 10.1139/cjm-2014-0087

Berger, C., Madshus, I. H., and Stang, E. (2012). Cetuximab in combination with anti-human IgG antibodies efficiently down-regulates the EGF receptor by macropinocytosis. Exp. Cell Res. 318, 2578-2591. doi: 10.1016/j.yexcr.2012.09.001

Bhattacharyya, J., Bellucci, J. J., Weitzhandler, I., McDaniel, J. R., Spasojevic, I., Li, X., et al. (2015). A paclitaxel-loaded recombinant polypeptide nanoparticle outperforms Abraxane in multiple murine cancer models. Nat. Commun. 6:7939. doi: 10.1038/ncomms8939

Biswas, S., Dodwadkar, N. S., Deshpande, P. P., Parab, S., and Torchilin, V. P. (2013). Surface functionalization of doxorubicin-loaded liposomes with octa-arginine for enhanced anticancer activity. Eur. J. Pharm. Biopharm. 84, 517-525. doi: 10.1016/j.ejpb.2012.12.021

Bohdanowicz, M., and Grinstein, S. (2013). Role of phospholipids in endocytosis, phagocytosis, and macropinocytosis. Physiol. Rev. 93, 69-106. doi: 10.1152/physrev.00002.2012

BoseDasgupta, S., Moes, S., Jenoe, P., and Pieters, J. (2015). Cytokine-induced macropinocytosis in macrophages is regulated by 14-3-3zeta through its interaction with serine-phosphorylated coronin 1. FEBS J. 282, 1167-1181. doi: 10.1111/febs.13214

Brown, F. D., Rozelle, A. L., Yin, H. L., Balla, T., and Donaldson, J. G. (2001). Phosphatidylinositol 4,5-bisphosphate and Arf6-regulated membrane traffic. J. Cell Biol. 154, 1007-1017. doi: 10.1083/jcb.200103107 available arsenal in selectively combating cancers. Additional studies in how different cancers utilize macropinocytosis to further disease progression could allow development of novel therapeutics against specific cancers. On this same note, it will be interesting to determine how macropinocytosis is differentially regulated across various tissues and cell types. Understanding how specific cell types can uniquely regulate macropinocytosis may pave way for novel therapeutics that can specifically target those cell types. Lastly, further research in how macropinocytosis is uniquely regulated from other endocytic pathways should facilitate targeted therapeutics without lending systemic toxicity stemming from broadly inhibiting all endocytic pathways.

\section{AUTHOR CONTRIBUTIONS}

$\mathrm{KH}$ wrote the manuscript, SB edited the manuscript, BL selected the review topic and edited the manuscript.

\section{ACKNOWLEDGMENTS}

The work is supported by grants from the National Institutes of Health/National Cancer Institute (R01 CA171315, R01 CA118919, and R01 CA129491).

Brzeska, H., Koech, H., Pridham, K. J., Korn, E. D., and Titus, M. A. (2016). Selective localization of myosin-I proteins in macropinosomes and actin waves. Cytoskeleton (Hoboken) 73, 68-82. doi: 10.1002/cm.21275

Canton, J., Schlam, D., Breuer, C., Gütschow, M., Glogauer, M., and Grinstein, S. (2016). Calcium-sensing receptors signal constitutive macropinocytosis and facilitate the uptake of NOD2 ligands in macrophages. Nat. Commun. 7:11284. doi: $10.1038 /$ ncomms 11284

Chakraborty, S., Veettil, M. V., Bottero, V., and Chandran, B. (2012). Kaposi's sarcoma-associated herpesvirus interacts with EphrinA2 receptor to amplify signaling essential for productive infection. Proc. Natl. Acad. Sci. U.S.A. 109, E1163-E1172. doi: 10.1073/pnas.1119592109

Chen, J. J., Bozza, W. P., Di, X., Zhang, Y., Hallett, W., and Zhang, B. (2014). $\mathrm{H}$-Ras regulation of TRAIL death receptor mediated apoptosis. Oncotarget 5, 5125-5137. doi: 10.18632/oncotarget.2091

Chen, L. M., Hobbie, S., and Galan, J. E. (1996). Requirement of CDC42 for Salmonella-induced cytoskeletal and nuclear responses. Science 274, 2115-2118.

Chung, J. J., Huber, T. B., Gödel, M., Jarad, G., Hartleben, B., Kwoh, C., et al. (2015). Albumin-associated free fatty acids induce macropinocytosis in podocytes. $J$. Clin. Invest. 125, 2307-2316. doi: 10.1172/JCI79641

Commisso, C., Davidson, S. M., Soydaner-Azeloglu, R. G., Parker, S. J., Kamphorst, J. J., Hackett, S., et al. (2013). Macropinocytosis of protein is an amino acid supply route in Ras-transformed cells. Nature 497, 633-637. doi: 10.1038 /nature 12138

Commisso, C., Flinn, R. J., and Bar-Sagi, D. (2014). Determining the macropinocytic index of cells through a quantitative image-based assay. Nat. Protoc. 9, 182-192. doi: 10.1038/nprot.2014.004

Crimeen-Irwin, B., Ellis, S., Christiansen, D., Ludford-Menting, M. J., Milland, J., Lanteri, M., et al. (2003). Ligand binding determines whether CD46 is internalized by clathrin-coated pits or macropinocytosis. J. Biol. Chem. 278, 46927-46937. doi: 10.1074/jbc.M308261200

de Carvalho, T. M., Barrias, E. S., and de Souza, W. (2015). Macropinocytosis: a pathway to protozoan infection. Front. Physiol. 6:106. doi: 10.3389/fphys.2015.00106

de Vries, E., Tscherne, D. M., Wienholts, M. J., Cobos-Jiménez, V., Scholte, F., Garcia-Sastre, A., et al. (2011). Dissection of the influenza A virus endocytic 
routes reveals macropinocytosis as an alternative entry pathway. PLoS Pathog. 7:e1001329. doi: 10.1371/journal.ppat.1001329

Doherty, G. J., and McMahon, H. T. (2009). Mechanisms of endocytosis. Annu. Rev. Biochem. 78, 857-902. doi: 10.1146/annurev.biochem.78.081307.110540

Donaldson, J. G., Porat-Shliom, N., and Cohen, L. A. (2009). Clathrin-independent endocytosis: a unique platform for cell signaling and PM remodeling. Cell. Signal. 21, 1-6. doi: 10.1016/j.cellsig.2008.06.020

Dowrick, P., Kenworthy, P., McCann, B., and Warn, R. (1993). Circular ruffle formation and closure lead to macropinocytosis in hepatocyte growth factor/scatter factor-treated cells. Eur. J. Cell Biol. 61, 44-53.

Drosopoulos, K. G., Roberts, M. L., Cermak, L., Sasazuki, T., Shirasawa, S., Andera, L., et al. (2005). Transformation by oncogenic RAS sensitizes human colon cells to TRAIL-induced apoptosis by up-regulating death receptor 4 and death receptor 5 through a MEK-dependent pathway. J. Biol. Chem. 280, 22856-22867. doi: 10.1074/jbc.M412483200

Francis, C. L., Ryan, T. A., Jones, B. D., Smith, S. J., and Falkow, S. (1993). Ruffles induced by Salmonella and other stimuli direct macropinocytosis of bacteria. Nature 364, 639-642.

Frenz, T., Grabski, E., Durán, V., Hozsa, C., Stepczynska, A., Furch, M., et al. (2015). Antigen presenting cell-selective drug delivery by glycandecorated nanocarriers. Eur. J. Pharm. Biopharm. 95(Pt A), 13-17. doi: 10.1016/j.ejpb.2015.02.008

Gimenez, M. C., Rodríguez Aguirre, J. F., Colombo, M. I., and Delgui, L. R. (2015). Infectious bursal disease virus uptake involves macropinocytosis and trafficking to early endosomes in a Rab5-dependent manner. Cell. Microbiol. 17, 988-1007. doi: $10.1111 / \mathrm{cmi} .12415$

Grimmer, S., van Deurs, B., and Sandvig, K. (2002). Membrane ruffling and macropinocytosis in A431 cells require cholesterol. J. Cell Sci. 115(Pt 14), 2953-2962.

Ha, K. D., Bidlingmaier, S. M., Zhang, Y., Su, Y., and Liu, B. (2014). High-content analysis of antibody phage-display library selection outputs identifies tumor selective macropinocytosis-dependent rapidly internalizing antibodies. Mol. Cell Proteomics 13, 3320-3331. doi: 10.1074/mcp.M114.039768

Hacker, U., Albrecht, R., and Maniak, M. (1997). Fluid-phase uptake by macropinocytosis in Dictyostelium. J. Cell Sci. 110 (Pt 2), 105-112.

Hall, J., Prabhakar, S., Balaj, L., Lai, C. P., Cerione, R. A., and Breakefield, X. O. (2016). Delivery of therapeutic proteins via extracellular vesicles: review and potential treatments for Parkinson's disease, glioma, and schwannoma. Cell. Mol. Neurobiol. 36, 417-427. doi: 10.1007/s10571-015-0309-0

Hansen, C. G., and Nichols, B. J. (2009). Molecular mechanisms of clathrin-independent endocytosis. J. Cell Sci. 122(Pt 11), 1713-1721. doi: $10.1242 /$ jcs. 033951

Hayashi, Y., Mizuno, R., Ikramy, K. A., Akita, H., and Harashima, H. (2012). Pretreatment of hepatocyte growth factor gene transfer mediated by octaarginine peptide-modified nanoparticles ameliorates LPS/Dgalactosamine-induced hepatitis. Nucleic Acid Ther. 22, 360-363. doi: 10.1089/nat.2012.0352

Hernáez, B., Guerra, M., Salas, M. L., and Andres, G. (2016). African swine fever virus undergoes outer envelope disruption, capsid disassembly and inner envelope fusion before core release from multivesicular endosomes. PLoS Pathog. 12:e1005595. doi: 10.1371/journal.ppat.1005595

Hetzenecker, S., Helenius, A., and Krzyzaniak, M. A. (2016). HCMV induces macropinocytosis for host cell entry in fibroblasts. Traffic 17, 351-368. doi: $10.1111 /$ tra. 12355

Heuser, J. (1989). The role of coated vesicles in recycling of synaptic vesicle membrane. Cell Biol. Int. Rep. 13, 1063-1076. doi: 10.1016/03091651(89)90020-9

Hewlett, L. J., Prescott, A. R., and Watts, C. (1994). The coated pit and macropinocytic pathways serve distinct endosome populations. J. Cell Biol. 124, 689-703. doi: $10.1083 /$ jcb.124.5.689

Holmes, B. B., DeVos, S. L., Kfoury, N., Li, M., Jacks, R., Yanamandra, K., et al. (2013). Heparan sulfate proteoglycans mediate internalization and propagation of specific proteopathic seeds. Proc. Natl. Acad. Sci. U.S.A. 110, E3138-E3147. doi: 10.1073/pnas.1301440110

Hunt, C. L., Kolokoltsov, A. A., Davey, R. A., and Maury, W. (2011). The Tyro3 receptor kinase Axl enhances macropinocytosis of Zaire ebolavirus. J. Virol. 85, 334-347. doi: 10.1128/JVI.01278-09
Iglesias, R., and Koria, P. (2015). Leveraging growth factor induced macropinocytosis for targeted treatment of lung cancer. Med. Oncol. 32:259. doi: 10.1007/s12032-015-0708-6

Innocenti, M., Gerboth, S., Rottner, K., Lai, F. P., Hertzog, M., Stradal, T. E., et al. (2005). Abil regulates the activity of N-WASP and WAVE in distinct actin-based processes. Nat. Cell Biol. 7, 969-976. doi: 10.1038/ncb1304

Innocenti, M., Zucconi, A., Disanza, A., Frittoli, E., Areces, L. B., Steffen, A., et al. (2004). Abil is essential for the formation and activation of a WAVE2 signalling complex. Nat. Cell Biol. 6, 319-327. doi: 10.1038/ncb1105

Ivanov, A. I. (2008). Pharmacological inhibition of endocytic pathways: is it specific enough to be useful? Methods Mol. Biol. 440, 15-33. doi: 10.1007/978-1-59745$178-9 \_2$

Javeed, N., Sagar, G., Dutta, S. K., Smyrk, T. C., Lau, J. S., Bhattacharya, S., et al. (2015). Pancreatic cancer-derived exosomes cause paraneoplastic beta-cell dysfunction. Clin. Cancer Res. 21, 1722-1733. doi: 10.1158/1078-0432.CCR-142022

Kamphorst, J. J., Nofal, M., Commisso, C., Hackett, S. R., Lu, W., Grabocka, E., et al. (2015). Human pancreatic cancer tumors are nutrient poor and tumor cells actively scavenge extracellular protein. Cancer Res. 75, 544-553. doi: 10.1158/0008-5472.CAN-14-2211

Kankaanpää, P., Tiitta, S., Bergman, L., Puranen, A. B., von Haartman, E., Linden, M., et al. (2015). Cellular recognition and macropinocytosis-like internalization of nanoparticles targeted to integrin alpha2beta1. Nanoscale 7, 17889-17901. doi: 10.1039/C5NR06218G

Kaushansky, A., Douglass, A. N., Arang, N., Vigdorovich, V., Dambrauskas, N., Kain, H. S., et al. (2015). Malaria parasites target the hepatocyte receptor EphA2 for successful host infection. Science 350, 1089-1092. doi: $10.1126 /$ science.aad 3318

Kerr, M. C., and Teasdale, R. D. (2009). Defining macropinocytosis. Traffic 10, 364-371. doi: 10.1111/j.1600-0854.2009.00878.x

Kettler, K., Veltman, K., van de Meent, D., van Wezel, A., and Hendriks, A. J. (2014). Cellular uptake of nanoparticles as determined by particle properties, experimental conditions, and cell type. Environ. Toxicol. Chem. 33, 481-492. doi: $10.1002 /$ etc. 2470

Khalil, I. A., Kogure, K., Futaki, S., Hama, S., Akita, H., Ueno, M., et al. (2007). Octaarginine-modified multifunctional envelope-type nanoparticles for gene delivery. Gene Ther. 14, 682-689. doi: 10.1038/sj.gt.3302910

Kimmelman, A. C. (2015). Metabolic dependencies in RAS-driven cancers. Clin. Cancer Res. 21, 1828-1834. doi: 10.1158/1078-0432.CCR-14-2425

Kitambi, S. S., Toledo, E. M., Usoskin, D., Wee, S., Harisankar, A., Svensson, R., et al. (2014). Vulnerability of glioblastoma cells to catastrophic vacuolization and death induced by a small molecule. Cell 157, 313-328. doi: 10.1016/j.cell.2014.02.021

Koivusalo, M., Welch, C., Hayashi, H., Scott, C. C., Kim, M., Alexander, T., et al. (2010). Amiloride inhibits macropinocytosis by lowering submembranous $\mathrm{pH}$ and preventing Rac1 and Cdc42 signaling. J. Cell Biol. 188, 547-563. doi: $10.1083 /$ jcb. 200908086

Koumakpayi, I. H., Le Page, C., Delvoye, N., Saad, F., and Mes-Masson, A. M. (2011). Macropinocytosis inhibitors and Arf6 regulate ErbB3 nuclear localization in prostate cancer cells. Mol. Carcinog. 50, 901-912. doi: $10.1002 / \mathrm{mc} .20766$

Kratz, F. (2008). Albumin as a drug carrier: design of prodrugs, drug conjugates and nanoparticles. J. Control. Release 132, 171-183. doi: 10.1016/j.jconrel.2008.05.010

Lanzetti, L., Palamidessi, A., Areces, L., Scita, G., and Di Fiore, P. P. (2004). Rab5 is a signalling GTPase involved in actin remodelling by receptor tyrosine kinases. Nature 429, 309-314. doi: 10.1038/nature02542

Levin, R., Grinstein, S., and Schlam, D. (2015). Phosphoinositides in phagocytosis and macropinocytosis. Biochim. Biophys. Acta 1851, 805-823. doi: 10.1016/j.bbalip.2014.09.005

Lewis, W. H. (1937). Pinocytosis by malignant cells. Am. J. Cancer 29, 666-679.

Li, C., Macdonald, J. I., Hryciw, T., and Meakin, S. O. (2010). Nerve growth factor activation of the TrkA receptor induces cell death, by macropinocytosis, in medulloblastoma Daoy cells. J. Neurochem. 112, 882-899. doi: 10.1111/j.14714159.2009.06507.x

Li, H. H., Li, J., Wasserloos, K. J., Wallace, C., Sullivan, M. G., Bauer, P. M., et al. (2013). Caveolae-dependent and -independent uptake of albumin 
in cultured rodent pulmonary endothelial cells. PLoS ONE 8:e81903. doi: 10.1371/journal.pone.0081903

Liang, C., Guo, B., Wu, H., Shao, N., Li, D., Liu, J., et al. (2015). Aptamerfunctionalized lipid nanoparticles targeting osteoblasts as a novel RNA interference-based bone anabolic strategy. Nat. Med. 21, 288-294. doi: 10.1038/nm.3791

Lim, J. P., and Gleeson, P. A. (2011). Macropinocytosis: an endocytic pathway for internalising large gulps. Immunol. Cell Biol. 89, 836-843. doi: 10.1038/icb.2011.20

Liu, X. L., Zhang, W. J., Wei, G., and Lu, W. Y. (2012). Poly(arginine)8 enhanced intestinal absorption of insulin-loaded nanoparticles. Yao Xиe Xиe Bao 47, $512-516$.

Liu, X., Wu, F., Tian, Y., Wu, M., Zhou, Q., Jiang, S., et al. (2016). Size dependent cellular uptake of rod-like bionanoparticles with different aspect ratios. Sci. Rep. 6, 24567. doi: 10.1038/srep24567

Liu, Y., Ran, R., Chen, J., Kuang, Q., Tang, J., Mei, L., et al. (2014). Paclitaxel loaded liposomes decorated with a multifunctional tandem peptide for glioma targeting. Biomaterials 35, 4835-4847. doi: 10.1016/j.biomaterials.2014.02.031

Mack, N. A., Whalley, H. J., Castillo-Lluva, S., and Malliri, A. (2011). The diverse roles of Rac signaling in tumorigenesis. Cell Cycle 10, 1571-1581. doi: $10.4161 /$ cc. 10.10 .15612

Magzoub, M., Sandgren, S., Lundberg, P., Oglecka, K., Lilja, J., Wittrup, A., et al. (2006). N-terminal peptides from unprocessed prion proteins enter cells by macropinocytosis. Biochem. Biophys. Res. Commun. 348, 379-385. doi: 10.1016/j.bbrc.2006.07.065

Maltese, W. A., and Overmeyer, J. H. (2015). Non-apoptotic cell death associated with perturbations of macropinocytosis. Front. Physiol. 6:38. doi: 10.3389/fphys.2015.00038

Mañes, S., Ana Lacalle, R., Gómez-Moutón, C., and Martinez, A. C. (2003). From rafts to crafts: membrane asymmetry in moving cells. Trends Immunol. 24, 320-326. doi: 10.1016/S1471-4906(03)00137-6

Mercer, J., and Helenius, A. (2008). Vaccinia virus uses macropinocytosis and apoptotic mimicry to enter host cells. Science 320, 531-535. doi: 10.1126/science. 1155164

Moody, P. R., Sayers, E. J., Magnusson, J. P., Alexander, C., Borri, P., Watson, P., et al. (2015). Receptor crosslinking: a general method to trigger internalization and lysosomal targeting of therapeutic receptor:ligand complexes. Mol. Ther. 23, 1888-1898. doi: 10.1038/mt.2015.178

Mullins, R. D., Heuser, J. A., and Pollard, T. D. (1998). The interaction of Arp2/3 complex with actin: nucleation, high affinity pointed end capping, and formation of branching networks of filaments. Proc. Natl. Acad. Sci. U.S.A. 95, 6181-6186. doi: 10.1073/pnas.95.11.6181

Munson, P., and Shukla, A. (2015). Exosomes: potential in cancer diagnosis and therapy. Medicines (Basel) 2, 310-327. doi: 10.3390/medicines 2040310

Naci, D., Vuori, K., and Aoudjit, F. (2015). Alpha2betal integrin in cancer development and chemoresistance. Semin. Cancer Biol. 35, 145-153. doi: 10.1016/j.semcancer.2015.08.004

Nakase, I., Kobayashi, N. B., Takatani-Nakase, T., and Yoshida, T. (2015). Active macropinocytosis induction by stimulation of epidermal growth factor receptor and oncogenic Ras expression potentiates cellular uptake efficacy of exosomes. Sci. Rep. 5:10300. doi: 10.1038/srep 10300

Nakase, I., Niwa, M., Takeuchi, T., Sonomura, K., Kawabata, N., Koike, Y., et al. (2004). Cellular uptake of arginine-rich peptides: roles for macropinocytosis and actin rearrangement. Mol. Ther. 10, 1011-1022. doi: 10.1016/j.ymthe.2004.08.010

Norbury, C. C., Hewlett, L. J., Prescott, A. R., Shastri, N., and Watts, C. (1995). Class I MHC presentation of exogenous soluble antigen via macropinocytosis in bone marrow macrophages. Immunity 3, 783-791. doi: 10.1016/10747613(95)90067-5

Northfelt, D. W., Allred, J. B., Liu, H., Hobday, T. J., Rodacker, M. W., Lyss, A. P., et al. (2014). Phase 2 trial of paclitaxel polyglumex with capecitabine for metastatic breast cancer. Am. J. Clin. Oncol. 37, 167-171. doi: 10.1097/COC.0b013e31826e0550

Ocaña-Morgner, C., Reichardt, P., Chopin, M., Braungart, S., Wahren, C., Gunzer, M., et al. (2011). Sphingosine 1-phosphate-induced motility and endocytosis of dendritic cells is regulated by SWAP-70 through RhoA. J. Immunol. 186, 5345-5355. doi: 10.4049/jimmunol.1003461
Orth, J. D., Krueger, E. W., Weller, S. G., and McNiven, M. A. (2006). A novel endocytic mechanism of epidermal growth factor receptor sequestration and internalization. Cancer Res. 66, 3603-3610. doi: 10.1158/0008-5472.CAN-052916

Overmeyer, J. H., Kaul, A., Johnson, E. E., and Maltese, W. A. (2008). Active ras triggers death in glioblastoma cells through hyperstimulation of macropinocytosis. Mol. Cancer Res. 6, 965-977. doi: 10.1158/1541-7786.MCR07-2036

Palm, W., Park, Y., Wright, K., Pavlova, N. N., Tuveson, D. A., and Thompson, C. B. (2015). The utilization of extracellular proteins as nutrients is suppressed by mTORC1. Cell 162, 259-270. doi: 10.1016/j.cell.2015.06.017

Pitt, J. M., André, F., Amigorena, S., Soria, J. C., Eggermont, A., Kroemer, G., et al. (2016). Dendritic cell-derived exosomes for cancer therapy. J. Clin. Invest. 126, 1224-1232. doi: 10.1172/JCI81137

Qian, Y., Wang, X., Liu, Y., Li, Y., Colvin, R. A., Tong, L., et al. (2014). Extracellular ATP is internalized by macropinocytosis and induces intracellular ATP increase and drug resistance in cancer cells. Cancer Lett. 351, 242-251. doi: 10.1016/j.canlet.2014.06.008

Qin, Y., Zhang, Q., Chen, H., Yuan, W., Kuai, R., Xie, F., et al. (2012). Comparison of four different peptides to enhance accumulation of liposomes into the brain. J. Drug Target. 20, 235-245. doi: 10.3109/1061186X.2011.639022

Racoosin, E. L., and Swanson, J. A. (1992). M-CSF-induced macropinocytosis increases solute endocytosis but not receptor-mediated endocytosis in mouse macrophages. J. Cell. Sci. 102(Pt 4), 867-880.

Racoosin, E. L., and Swanson, J. A. (1993). Macropinosome maturation and fusion with tubular lysosomes in macrophages. J. Cell Biol. 121, 1011-1020. doi: 10.1083/jcb.121.5.1011

Radhakrishna, H., Klausner, R. D., and Donaldson, J. G. (1996). Aluminum fluoride stimulates surface protrusions in cells overexpressing the ARF6 GTPase. J. Cell Biol. 134, 935-947. doi: 10.1083/jcb.134.4.935

Redelman-Sidi, G., Iyer, G., Solit, D. B., and Glickman, M. S. (2013). Oncogenic activation of Pak1-dependent pathway of macropinocytosis determines BCG entry into bladder cancer cells. Cancer Res. 73, 1156-1167. doi: 10.1158/00085472.CAN-12-1882

Reif, R., Adawy, A., Vartak, N., Schröder, J., Gunther, G., Ghallab, A., et al. (2016). Activated ErbB3 translocates to the nucleus via Clathrin-independent endocytosis, which is associated with proliferating cells. J. Biol. Chem. 291, 3837-3847. doi: 10.1074/jbc.M115.686782

Reyes-Reyes, E. M., Teng, Y., and Bates, P. J. (2010). A new paradigm for aptamer therapeutic AS1411 action: uptake by macropinocytosis and its stimulation by a nucleolin-dependent mechanism. Cancer Res. 70, 8617-8629. doi: 10.1158/0008-5472.CAN-10-0920

Schliwa, M. (1982). Action of cytochalasin D on cytoskeletal networks. J. Cell Biol. 92, 79-91. doi: $10.1083 /$ jcb.92.1.79

Schmees, C., Villaseñor, R., Zheng, W., Ma, H., Zerial, M., Heldin, C. H., et al. (2012). Macropinocytosis of the PDGF beta-receptor promotes fibroblast transformation by H-RasG12V. Mol. Biol. Cell 23, 2571-2582. doi: 10.1091/mbc.E11-04-0317

Schnatwinkel, C., Christoforidis, S., Lindsay, M. R., Uttenweiler-Joseph, S., Wilm, M., Parton, R. G., et al. (2004). The Rab5 effector Rabankyrin-5 regulates and coordinates different endocytic mechanisms. PLoS Biol. 2:E261. doi: 10.1371/journal.pbio.0020261

Seastone, D. J., Harris, E., Temesvari, L. A., Bear, J. E., Saxe, C. L., and Cardelli, J. (2001). The WASp-like protein scar regulates macropinocytosis, phagocytosis and endosomal membrane flow in Dictyostelium. J. Cell Sci. 114(Pt 14), 2673-2683.

Selwan, E. M., Finicle, B. T., Kim, S. M., and Edinger, A. L. (2016). Attacking the supply wagons to starve cancer cells to death. FEBS Lett. 590, 885-907. doi: $10.1002 / 1873-3468.12121$

Shi, Y., Su, C., Cui, W., Li, H., Liu, L., Feng, B., et al. (2014). Gefitinib loaded folate decorated bovine serum albumin conjugated carboxymethyl-betacyclodextrin nanoparticles enhance drug delivery and attenuate autophagy in folate receptor-positive cancer cells. J. Nanobiotechnology 12:43. doi: 10.1186/s12951-014-0043-7

Simonsen, A., Wurmser, A. E., Emr, S. D., and Stenmark, H. (2001). The role of phosphoinositides in membrane transport. Curr. Opin. Cell Biol. 13, 485-492. doi: 10.1016/S0955-0674(00)00240-4 
Stephen, A. G., Esposito, D., Bagni, R. K., and McCormick, F. (2014). Dragging ras back in the ring. Cancer Cell 25, 272-281. doi: 10.1016/j.ccr.2014.02.017

Tanaka, G., Nakase, I., Fukuda, Y., Masuda, R., Oishi, S., Shimura, K., et al. (2012). CXCR4 stimulates macropinocytosis: implications for cellular uptake of arginine-rich cell-penetrating peptides and HIV. Chem. Biol. 19, 1437-1446. doi: 10.1016/j.chembiol.2012.09.011

Tang, W., Tam, J. H., Seah, C., Chiu, J., Tyrer, A., Cregan, S. P., et al. (2015). Arf6 controls beta-amyloid production by regulating macropinocytosis of the Amyloid Precursor Protein to lysosomes. Mol. Brain 8:41. doi: 10.1186/s13041015-0129-7

Teranishi, F., Takahashi, N., Gao, N., Akamo, Y., Takeyama, H., Manabe, T., et al. (2009). Phosphoinositide 3-kinase inhibitor (wortmannin) inhibits pancreatic cancer cell motility and migration induced by hyaluronan in vitro and peritoneal metastasis in vivo. Cancer Sci. 100, 770-777. doi: 10.1111/j.13497006.2009.01084.x

Thapa, N., Choi, S., Hedman, A., Tan, X., and Anderson, R. A. (2013). Phosphatidylinositol phosphate 5-kinase Igammai2 in association with Src controls anchorage-independent growth of tumor cells. J. Biol. Chem. 288, 34707-34718. doi: 10.1074/jbc.M113.512848

Tkach, M., and Thery, C. (2016). Communication by extracellular vesicles: where we are and where we need to go. Cell 164, 1226-1232. doi: 10.1016/j.cell.2016.01.043

Viaud, J., Mansour, R., Antkowiak, A., Mujalli, A., Valet, C., Chicanne, G., et al. (2016). Phosphoinositides: important lipids in the coordination of cell dynamics. Biochimie 125, 250-258. doi: 10.1016/j.biochi.2015.09.005

Walsh, M., Tangney, M., O’Neill, M. J., Larkin, J. O., Soden, D. M., McKenna, S. L., et al. (2006). Evaluation of cellular uptake and gene transfer efficiency of pegylated poly-L-lysine compacted DNA: implications for cancer gene therapy. Mol. Pharm. 3, 644-653. doi: 10.1021/mp0600034

Wang, H., Yang, T., and Wu, X. (2015). 5-Fluorouracil preferentially sensitizes mutant KRAS non-small cell lung carcinoma cells to TRAIL-induced apoptosis. Mol. Oncol. 9, 1815-1824. doi: 10.1016/j.molonc.2015.06.003

West, M. A., Bretscher, M. S., and Watts, C. (1989). Distinct endocytotic pathways in epidermal growth factor-stimulated human carcinoma A431 cells. J. Cell Biol. 109(6 Pt 1), 2731-2739. doi: 10.1083/jcb.109.6.2731

Whiteside, T. L. (2016). Tumor-derived exosomes and their role in cancer progression. Adv. Clin. Chem. 74, 103-141. doi: 10.1016/bs.acc.2015.12.005

Wolpin, B. M., Rubinson, D. A., Wang, X., Chan, J. A., Cleary, J. M., Enzinger, P. C., et al. (2014). Phase II and pharmacodynamic study of autophagy inhibition using hydroxychloroquine in patients with metastatic pancreatic adenocarcinoma. Oncologist 19, 637-638. doi: 10.1634/theoncologist.20140086
Wu, Q., Wang, H., Zhao, X., Shi, Y., Jin, M., Wan, B., et al. (2013). Identification of G-protein-coupled receptor 120 as a tumor-promoting receptor that induces angiogenesis and migration in human colorectal carcinoma. Oncogene 32, 5541-5550. doi: 10.1038/onc.2013.264

Yoshida, S., Pacitto, R., Yao, Y., Inoki, K., and Swanson, J. A. (2015). Growth factor signaling to mTORC1 by amino acid-laden macropinosomes. J. Cell Biol. 211, 159-172. doi: 10.1083/jcb.201504097

Zaro, J. L., Rajapaksa, T. E., Okamoto, C. T., and Shen, W. C. (2006). Membrane transduction of oligoarginine in HeLa cells is not mediated by macropinocytosis. Mol. Pharm. 3, 181-186. doi: 10.1021/mp0500869

Zeineddine, R., and Yerbury, J. J. (2015). The role of macropinocytosis in the propagation of protein aggregation associated with neurodegenerative diseases. Front. Physiol. 6, 277. doi: 10.3389/fphys.2015.00277

Zeitouni, D., Pylayeva-Gupta, Y., Der, C. J., and Bryant, K. L. (2016). KRAS mutant pancreatic cancer: no lone path to an effective treatment. Cancers (Basel) 8:45. doi: 10.3390/cancers 8040045

Zhang, C., Tang, N., Liu, X., Liang, W., Xu, W., and Torchilin, V. P. (2006). siRNAcontaining liposomes modified with polyarginine effectively silence the targeted gene. J. Control. Release 112, 229-239. doi: 10.1016/j.jconrel.2006.01.022

Zhang, Y., Li, L., Han, M., Hu, J., and Zhang, L. (2015). Amphiphilic lipopeptidemediated transport of insulin and cell membrane penetration mechanism. Molecules 20, 21569-21583. doi: 10.3390/molecules201219771

Zhao, H., Yang, L., Baddour, J., Achreja, A., Bernard, V., Moss, T., et al. (2016). Tumor microenvironment derived exosomes pleiotropically modulate cancer cell metabolism. Elife 5:e10250. doi: 10.7554/eLife.10250

Zhao, M., Lei, C., Yang, Y., Bu, X., Ma, H., Gong, H., et al. (2015a). Abraxane, the nanoparticle formulation of paclitaxel can induce drug resistance by upregulation of P-gp. PLoS ONE 10:e0131429. doi: 10.1371/journal.pone.0131429

Zhao, M., Li, H., Bu, X., Lei, C., Fang, Q., and Hu, Z. (2015b). Quantitative proteomic analysis of cellular resistance to the nanoparticle abraxane. ACS Nano 9, 10099-10112. doi: 10.1021/acsnano.5b03677

Conflict of Interest Statement: The authors declare that the research was conducted in the absence of any commercial or financial relationships that could be construed as a potential conflict of interest.

Copyright (c) $2016 \mathrm{Ha}$, Bidlingmaier and Liu. This is an open-access article distributed under the terms of the Creative Commons Attribution License (CC BY). The use, distribution or reproduction in other forums is permitted, provided the original author(s) or licensor are credited and that the original publication in this journal is cited, in accordance with accepted academic practice. No use, distribution or reproduction is permitted which does not comply with these terms. 